\title{
Bupivacaine Continuous Spinal Analgesia Induced Aseptic Meningitis in Systemic Sclerosis Patient: A Case Report
}

\author{
Delfino L, Olivieri B, Tinazzi E, Caminati M and Lunardi C* \\ Department of Medicine, University of Verona, Italy \\ *Corresponding author: Lunardi C, Department of Medicine, University of Verona, Italy
}

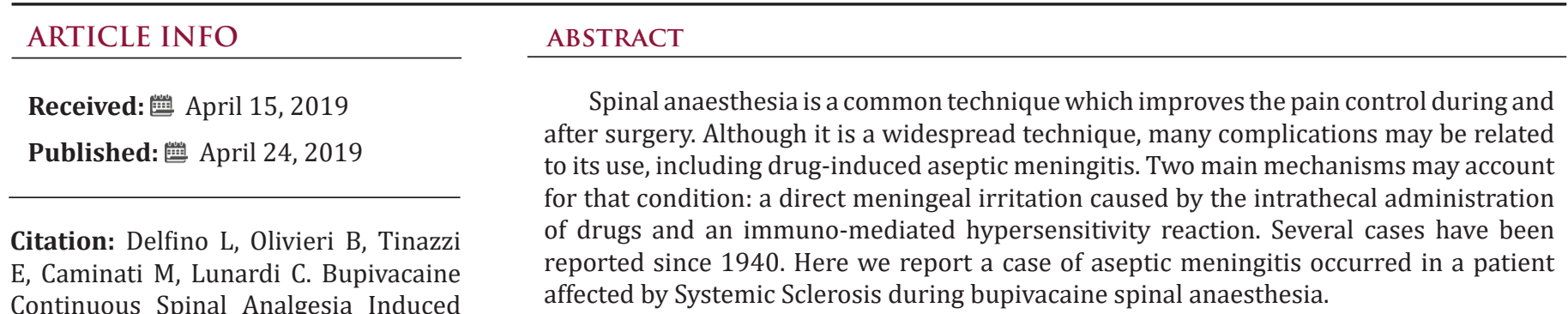

Keywords: Systemic Sclerosis; Drug-Induced Aseptic Meningitis; Bupivacaine; Spinal Anaesthesia

Abbreviations: DIAM: Drug Induced Aseptic Meningitis; CSF: Cerebrospinal Fluid; PCR: Polymerase Chain Reaction; GCS: Glasgow Coma Scale

\section{Introduction}

Spinal anaesthesia is a very common technique which surely improves the pain control during and after surgical procedures. Continuous spinal analgesia offers relevant advantages. It allows to reduce the recovery period and to decrease anesthetic concentration, minimizing its potential adverse effects including cardiovascular instability [1]. Nevertheless, it is not free from complications, such as the risk of catheter dislocation, headache, neurological injury, infections. It has been reported that spinal anaesthesia may also cause aseptic meningitis, which is a rare but serious condition [1,2].

Several drugs, including NSAID, Immunoglobulins and Bupivacaine may be responsible for drug-induced aseptic meningitis (DIAM). Two main mechanisms account for it: a direct meningeal irritation caused by the intrathecal administration of drugs and an immuno-mediated hypersensitivity reaction, especially in patients affected by autoimmune diseases. Secondary encephalitis has been also observed $[3,4]$. DIAM is a diagnosis of exclusion and represents a completely reversible condition after few days from the drug interruption. Here we report a case of Bupivacaine-induced aseptic meningitis in a patient affected by Systemic Sclerosis.

\section{Case Description}

A 65 years old patient, affected by Systemic Sclerosis, was admitted to our Autoimmune Diseases Unit for a critical ischemia of the right foot. His medical history included the amputation of I-II-III e IV toes of the left foot for ischemic necrosis, interstitial lung disease, autoimmune hepatitis and hiatal hernia with Barrett's esophagus. Despite the medical treatment, including prostanoid combined with antiplatelet and anticoagulant therapy, and an endovascular attempt of revascularization, below-knee amputation was needed. Under all aseptic precautions, the lumbar area was prepared using povidone iodine $10 \%$ and a sterile drape was placed; bupivacaine $0,1 \%(5 \mathrm{ml})$ was administrated in L3-L4 interspace. The surgery was carried out without complications and the patient did not present any hemodynamic or respiratory events. In order to control post-operative pain, a lumbar epidural catheter with continuous infusion of Bupivacaine $0,1 \%$ was placed.

After 48 hours, the patient showed mental state alteration including agitated behavior, confusion and loss of consciousness. He had fever $\left(38.5^{\circ} \mathrm{C}\right)$ but no focal neurological deficits were recorded. Blood pressure was $140 / 70 \mathrm{mmHg}$. Laboratory analysis revealed 
a hemoglobin concentration at $9.7 \mathrm{~g} / \mathrm{dL}$, not differently from the previous days, WBC $16150 / \mathrm{mm}^{3}$ with $14000 / \mathrm{mm}^{3}$ neutrophils and platelets $286.000 / \mathrm{mm}^{3} \mathrm{C}$-reactive protein was $100 \mathrm{mg} / \mathrm{L}$ and procalcitonin was negative. Renal function tests, serum electrolytes and ammonia blood test were within normal limits. The lumbar epidural catheter was removed, and, in the suspicion of a septic status, a wide spectrum antibiotic therapy was started. Since the neurological status was very compromised a brain CT scan and MRI were performed. The CT scan did not show anomalies, while the MRI detected a diffuse enhancement of the pachymeninx (Figure 1). The EEG revealed a diffuse and aspecific electrical disorder. A diagnostic lumbar puncture displayed a limpid cerebrospinal fluid (CSF), with normal pressure, and the laboratory analysis showed a protein concentration of $2,73 \mathrm{~g} / \mathrm{L}$, a glucose concentration of 1.8 $\mathrm{mmol} / \mathrm{L}$ and leukocytes 10/microL.

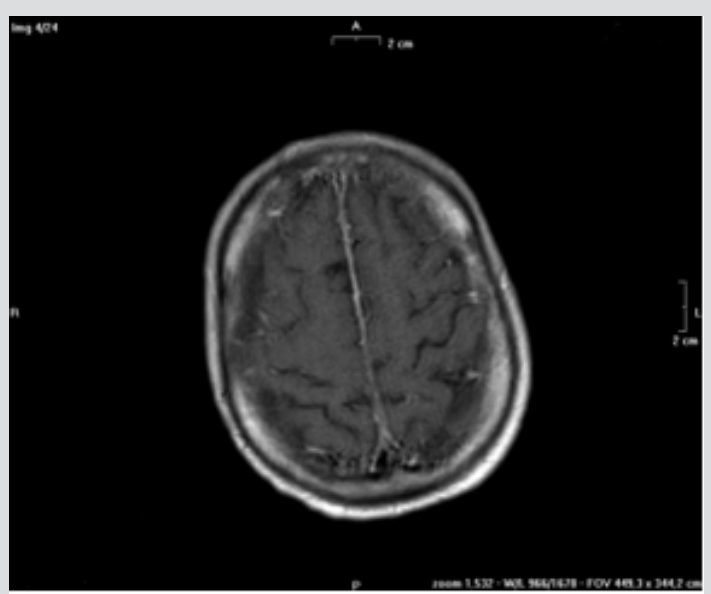

Figure 1: Brain MRI section after contrast administration showed a diffuse signal enhancement of the pachymeninx.

The bacterial cultures were negative and also the polymerase chain reaction (PCR) of the CSF was negative for all the verified microbic genome sequences (HSV1, HSV2, HHV6, HHV8, EBV, CMV, VZV, Human Parechovirus, enterovirus, E.coli, Hemophilus Influenzae, Listeria, Neisseria Meningitidis, Streptococcus Agalactiae, Streptococcus Pneumoniae, Cryptococcus Neoformans and Gatii). Also, the blood and urine microbiological tests did not reveal any bacterial growth. The patient experienced rapid worsening of the state of consciousness with a Glasgow Coma Scale (GCS) of 3 out of 15 points, together with neck rigidity, so he was moved to the Intensive Care Unit. The patient was diagnosed with aseptic meningitis. 48 hours after the symptom's onset and the suspension of bupivacaine administration, the patient was awake, afebrile, but a mild mental confusion status was observed during the following week. The patient finally experienced a complete remission, without neurological deficits.

\section{Discussion}

To the best of our knowledge, here we have reported for the first time a case of aseptic meningitis related to bupivacaine spinal analgesia administration in a patient with Systemic Sclerosis. According to the data reported above, drug-induced aseptic meningitis is a very rare adverse reaction to several drugs and is diagnosed by exclusion. As already described in literature, clinical manifestations during a drug-induce aseptic meningitis are very aspecific. Fever, headache, confusion, agitation, a stupor or a comatose status may represent the main manifestations, without any common sign of meningeal irritation or photophobia. Neuroimaging is usually noninformative; sometimes Brain MRI may show a diffuse hyper intensity of leptomeningeal spaces $[2,5,6]$. Cerebro-spinal fluid analysis usually show pleocytosis (hundred to several thousand cells per microliter). Polymorphonuclear predominance is very common, but lymphocytes and eosinophilic presence have also been reported. Moreover, the proteins levels are usually elevated, whereas the concomitant glucose levels remain normal. Of course, bacterial cultures and viral genome research are negative [4-7].

A spontaneous recovery within few days after drug discontinuation is helpful in the diagnosis [8]. In several cases, spinal anaesthesia with bupivacaine was performed [9]. The real pathogenetic mechanisms are still unclear but, in some cases, an immunologic T-cell mediated hypersensitivity reaction has been supposed specially in subjects affected by autoimmune disease. Indeed, the number of reports associating autoimmune conditions, mainly Systemic Lupus Erythematosus, with drug-induced aseptic meningitis is not negligible [10]. Our case report remarks the high risk of adverse drug reactions in patients with autoimmune diseases, probably due to an intrinsic immune-mediated susceptibility.

\section{References}

1. Kasai T, Yaegashi K, Hirose M, Fujita T, Tanaka Y (2003) Aseptic meningitis during combined continuous spinal and epidural analgesia. Acta Anaesthesiol Scand 47(6): 775-776.

2. Tateno F, Sakakibara R, Kishi M, Ogawa E (2010) Bupivacaine-induced chemical meningitis. J Neurol 257(8): 1327-1329.

3. Besocke AG, Santamarina R, Romano LM, Femminini RA (2007) Bupivacaine induced aseptic meningitis. Neurologia 22(8): 551-552.

4. Yelehe Okouma M, Czmil Garon J, Pape E, Petitpain N, Gillet P (2018) Drug-induced aseptic meningitis: a mini-review. Fundam Clin Pharmacol 32(3): 252-260.

5. Doghmi N, Meskine A, Benakroute A, Bensghir M, Baite A, et al. (2017) Aseptic meningitis following a bupivacaine spinal anesthesia. Pan Afr Med J 27: 192. 
6. Celik M, Kizilkaya M, Dostbil A, Dogan N, Parlak M, et al. (2014) Meningitis following spinal anaesthesia in an obstetric patient. Trop Doct 44(3): 179-181

7. Jarrin I, Sellier P, Lopes A, Morgand M, Makovec T, et al. (2016) Etiologies and Management of Aseptic Meningitis in Patients Admitted to an Internal Medicine Department. Medicine (Baltimore) 95(2): e2372.

8. Jolles S, Sewell WA, Leighton C (2000) Drug-induced aseptic meningitis: diagnosis and management. Drug Saf 22(3): 215-226.

\section{ISSN: 2574-1241}

DOI: 10.26717/BJSTR.2019.17.002995

Lunardi C. Biomed J Sci \& Tech Res

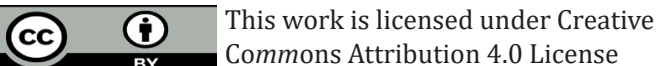

Submission Link: https://biomedres.us/submit-manuscript.php
9. Ducornet A, Brousous F, Jacob C, Egreteau PY, Tonnelier JM (2014) Meningitis after spinal anesthesia: think about bupivacaine! Ann Fr Anesth Reanim 33(4): 288-290.

10. Castagna J, Nosbaum A, Vial T, Rozieres A, Hacard F, et al. (2018) Druginduced aseptic meningitis: A possible T-cell-mediated hypersensitivity. J Allergy Clin Immunol Pract 6(4): 1409-1411.

\begin{tabular}{ll}
\hline $\begin{array}{l}\text { BIOMEDICAL } \\
\text { RESEARCHES }\end{array}$ & Assets of Publishing with us \\
\hline IsSN: $2574-1241$ & - Immediate, unrestricted online access \\
\end{tabular}

